\title{
Whole-sentence definition versus definição por genus proximum + differentiae specificae: um contraste entre duas técnicas definitórias
}

\author{
Whole-sentence definition versus analytical definition: a \\ contrast between two definitional techniques
}

\author{
Virginia Sita Farias \\ UFRGS
}

\begin{abstract}
The definition plays a major role within the microstructure of semasiological dictionaries. However, in spite of the lexicographer's interest in this subject, there is not a definitive solution yet. This paper aims at establishing a contrast between two definitional techniques: the whole-sentence definition and the analytical definition. Firstly, we presented a exhaustive analysis of whole-sentence definitions of nouns, with special attention to prototypicality effect in these definitions. Next, we compared the whole-sentence definitions with the analytical definitions in order to detect whether or not there is a significant difference between the results obtained with the use of one technique or another, at least regarding the definition of nouns.
\end{abstract}

Keywords

Definitional technique; whole-sentence definition; analytical definition. 


\section{Resumo}

A definição cumpre a função mais importante na microestrutura de um dicionário semasiológico. Contudo, apesar do interesse dos lexicógrafos por este assunto, ainda não foram produzidos estudos conclusivos sobre como gerar uma boa definição. Este artigo almeja estabelecer um contraste entre duas diferentes técnicas definitórias: a whole-sentence definition e a definição por genus proximum + differentiae specificae. Primeiramente, apresentamos uma análise exaustiva de definições de substantivos através da técnica de wholesentence definition, com atenção especial à representação da prototipicidade neste tipo de definição. A seguir, propusemos uma comparação com as paráfrases por genus proximum + differentiae specificae, a fim de detectar se há uma diferença significativa entre os resultados obtidos pelo emprego de uma ou outra técnica, pelo menos no que se refere à definição de substantivos.

\section{Palavras-chave}

Técnica definitória; whole-sentence definition; definição por genus proximum + differentiae specificae. 


\section{Introdução}

ssumindo que o dicionário semasiológico ${ }^{1}$ é um instrumento usado
principalmente para esclarecer dúvidas acerca da significação das palavras,
podemos dizer que as paráfrases definidoras cumprem a função mais importante na microestrutura ${ }^{2}$ desse tipo de obra. Isso justifica o fato de que a definição lexicográfica seja matéria de discussão em diversos trabalhos, a exemplo de Dubois e Dubois (1971, p. 84-89), Haensch (1982, p. 259-328), Seco (1987, p. 15-45), Martínez de Souza (1995, s.v. definición lingüística), Landau (2001, p. 153-216), Jackson (2002, p. 86-100), Beneduzi (2004), Beneduzi e Bugueño (2005), Beneduzi, Bugueño e Farias (2005) e Zanatta (2006). Contudo, apesar da importância atribuída à paráfrase definidora dentro da microestrutura do dicionário semasiológico e, consequentemente, de este ser um tema nuclear no âmbito da Lexicografia, ainda dispomos de poucos estudos conclusivos sobre como gerar uma boa definição, ou uma definição satisfatória (cf. BUGUEÑO, 2007b).

A primeira pergunta com a qual o lexicógrafo se vê confrontado diante da tarefa de definir uma unidade léxica é qual técnica explanatória empregar, considerando a diversidade de opções metodológicas de que a teoria metalexicográfica dispõe. ${ }^{3}$ Em atenção a esse fenômeno é que Bugueño (2007b) propõe uma taxonomia de paráfrases definidoras, formulada com base em dois parâmetros: 1) a perspectiva do ato da comunicação; 2) a metalinguagem empregada. ${ }^{4}$ De acordo com esse autor, uma tipologia é uma das variáveis que, combinada com uma sintaxe da definição e com um modelo semântico, permite chegar a uma paráfrase definidora realmente elucidativa para o consulente.

Na vanguarda das principais opções metodológicas disponíveis, está a definição por genus proximum + differentiae specificae, também chamada de definição aristotélica, definição hiperonímica, definição inclusiva (cf. MARTÍNEZ DE SOUZA, 1995, s.v.), ou ainda definição analítica (cf. GEERAERTS, 2003, p. 89 e LEW; DZIEMIANKO, 2006a e 2006b). Esse tipo de paráfrase é composta por um termo descritor, que é um hiperônimo da 
unidade definida (genus proximum), e um ou mais especificadores que cumprem a função de caracterizar a unidade definida, diferenciando-a dos seus cohipônimos (differentiae specificae), como podemos ver no exemplo a seguir:

ônibus $[\ldots]$ 1. Veículo automóvel para transporte público de passageiros, com itinerário preestabelecido. $(\mathrm{Au}, 1999 \text {, s.v. })^{5}$

Em Beneduzi (2004) e Beneduzi, Bugueño e Farias (2005), expuseram-se parâmetros para a redação das paráfrases definidoras em um dicionário de falsos amigos espanhol-português. ${ }^{6}$ Tais parâmetros, gerados com vistas a otimizar os resultados obtidos na redação das paráfrases, foram elaborados com base nos critérios de concisão, abrangência e circularidade, ${ }^{7}$ propostos por Martínez de Souza (1995, s.v. definición lingüística), e no princípio de submissão à prova da substituição, exposto em Seco (1987, p. 19-34). Cabe também citar Landau (2001, p. 157-171), que, por sua vez, apresenta boa parte dos critérios e princípios anteriormente discutidos por Seco (1987) e Martínez de Souza (1995) em forma de regras a serem observadas na elaboração das definições.

Além da definição por genus proximum + differentiae specificae, aludiremos, na presente ocasião, a um outro tipo de definição, chamada wholesentence definition (cf. HARTMANN; JAMES, 2001, s.v. definition style), definição sentencial (cf. GEERAERTS, 2003, p. 91), ou ainda definição contextual (cf. LEW; DZIEMIANKO, 2006a e 2006b). A whole-sentence definition normalmente conforma um período composto por duas orações: a primeira coloca a palavra-entrada em um contexto, e a segunda apresenta a definição propriamente dita (cf. LANDAU, 2001, p. 164 e LEW; DZIEMIANKO, 2006a). A estrutura desse tipo de definição inviabiliza a prova da substituição, que, por sua vez, é um dos princípios de redação das paráfrases por genus proximum + differentiae specificae (cf. LANDAU, 2001, p. 164-166). ${ }^{8}$ Observemos o seguinte exemplo:

love [...] 1 If you love someone, you feel romantically or sexually attracted to them, and they are very important to you. (CCLD 2003, s.v.)

Além da distinção formal verificada entre as paráfrases por genus proximum + differentiae specificae e as whole-sentence definitions, estas 
últimas ainda se distinguem das primeiras porque não somente definem a palavraentrada, mas mostram para o consulente o efeito de sentido provocado pelo uso do lexema definido. De acordo com Landau (2001), "[t]his style of defining [whole-sentence definition] has the virtue of immediately putting the usage in a social context as an interaction between people, whereas a traditional defining style is more abstract and less satisfactory" (LANDAU, 2001, p. 179). A seguir, apresentamos dois exemplos:

cute [...] 2 If you describe someone as cute, you think they are sexually attractive. (mainly AM INFORMAL) (CCLD, 2003, s.v.)

polite [...] 2 You can refer to people who consider themselves to be socially superior and to set standards of behaviour for everyone else as polite society or polite company. (CCLD, 2003, s.v.)

Essa característica das whole-sentence definitions fica evidente se comparamos as paráfrases transcritas acima com as definições tradicionais relacionadas abaixo:

cute [...] 2 (informal, especially NAmE) sexually attractive. (OALD, 2005, s.v.)

polite [...] $\mathbf{3}$ [only before noun] from a class of society that believes it is better than others. (OALD, 2005, s.v.)

Entretanto, a condição de superioridade das whole-sentence definitions sobre as demais definições não se verifica sempre. Comparemos, por exemplo, as definições de big extraídas, respectivamente, de CCLD (2003) e OALD (2005):

big [...] $1 \mathrm{~A}$ big person or thing is large in physical size. (CCLD, 2003, s.v.)

big [...] 1 large in size, degree, amount, etc. (OALD, 2005, s.v.)

Como se vê, salvo pelo fato de que a definição extraída de CCLD (2003) apresenta a palavra-entrada num contexto, informando ao consulente, neste caso, as classes de palavras às quais o adjetivo em questão pode servir de atributo, não há diferença, no que concerne à expressão do conteúdo semântico, entre as 
duas definições. Ademais, a vantagem da definição apresentada em CCLD (2003) poderia ser, se não suplantada, pelo menos equiparada em OALD (2005), se este dicionário apresentasse a atribuição do adjetivo antes da definição, entre parênteses ou colchetes, de modo a marcar a separação desta informação, que é parte do comentário de forma, do conteúdo da definição (comentário semântico). Assim, a referida obra poderia trazer uma paráfrase redigida da seguinte maneira: "[person or thing] large in size, degree, amount, etc."

Ressaltamos, ainda, que autores como Landau (2001, p. 180) também reconhecem que as whole-sentence definitions têm suas limitações: elas não seriam uma boa ferramenta para a definição de alguns substantivos, funcionando melhor na definição de verbos e de muitos adjetivos e advérbios. Neste trabalho, pois, nosso objetivo é, através de uma análise detalhada das definições, especialmente de substantivos, apresentadas em CCLD (2003) e em CcLD (2004), apontar as possíveis vantagens, bem como as restrições desta técnica de definição, propondo uma comparação com as paráfrases por genus proximum + differentiae specificae. Além disso, procuraremos oferecer explicações plausíveis para as limitações que detectarmos.

\section{Análise das whole-sentence definitions}

Uma das características mais peculiares das whole-sentence definitions é o seu viés extensional, que, no caso das definições de substantivos, está frequentemente representado pela presença de um elemento prototípico na sentença definidora (cf. 2.1.). Sendo assim, a análise à qual procederemos nesta seção será dividida em duas partes: na primeira, tentaremos mostrar como se dá a prototipicidade neste tipo de definição, com suas vantagens e suas falhas; na segunda, apresentaremos casos em que essa característica não está presente nas definições, seja por uma falha na redação delas, o que não é algo incomum na maioria das obras lexicográficas, seja por uma limitação da própria técnica definitória.

\subsection{A representação da prototipicidade nas whole-sentence definitions}

Na representação da prototipicidade verificada na redação das wholesentence definitions, é possível reconhecer uma correlação entre esta técnica 
de definição e a Teoria dos Protótipos. A referida teoria encontra sua base nos estudos da psicóloga Eleanor Rosh, tendo nascido como uma reação ao modelo das condições necessárias e suficientes ${ }^{9}$ (cf. LAKOFF, 1987, p. 39-57). Os primeiros trabalhos de Rosh demonstraram que as categorias não são compostas por membros detentores do mesmo status, como preconizava a teoria das condições necessárias e suficientes, mas, ao contrário, há membros dentro de uma categoria que são julgados como mais centrais que outros. Essa assimetria, chamada de efeito de centralidade ou de prototipicidade, pode ser exemplificada pela categoria mamífero, da qual vaca e cabra são membros mais prototípicos do que baleia e morcego. O protótipo é, pois, o membro considerado como o mais representativo de uma categoria. ${ }^{10}$ Dessa forma, pode-se dizer, grosso modo, que, enquanto uma paráfrase por genus proximum + differentiae specificae encontra seu fundamento no modelo das condições necessárias e suficientes, a Teoria dos Protótipos subjaz à técnica das whole-sentence definitions.

Segundo Geeraerts (2001, p. 13-16), o efeito de prototipicidade pode estar representado de duas maneiras distintas nas definições: 1) através da enumeração dos membros mais típicos de uma categoria, e 2) através da identificação dos traços mais prototípicos de uma classe.

\subsubsection{Enumeração dos membros mais típicos da categoria}

Antes de procedermos à análise, faz-se necessário que nos detenhamos em uma distinção fundamental proposta entre intensão (nível do significado) e extensão (nível do referente). A intensão é o conjunto de traços que caracterizam uma determinada entidade, de modo que a definição intensionalmente bem formulada é a que enumera os principais semas de determinada unidade léxica. A extensão, por outro lado, refere-se aos membros incluídos em uma determinada categoria. ${ }^{11}$ Uma definição que enumera os membros mais típicos da categoria é, pois, uma definição de caráter extensional. ${ }^{12}$ A enumeração é uma das estratégias utilizadas pelas whole-sentence definitions, como comprovamos através dos exemplos a seguir:

clothes $[\ldots]$ Clothes are the things that people wear, such as shirts, coats, trousers, and dresses. (CCLD, 2003, s.v.) 
drink [...] 5 Drink is alcohol, such as beer, wine, or whisky. (CCLD, 2003, s.v.)

human being [...] A human being is a man, woman, or child. (CCLD, 2003, s.v.)

vegetation [...] Vegetation is plants, trees and flowers; FORMAL. (CcLD, 2004, s.v.)

Como podemos ver, essas definições diferem essencialmente das paráfrases por genus proximum + differentiae specificae por utilizarem uma enumeração dos membros mais típicos da categoria (extensão), em detrimento da listagem de um conjunto de traços (semas) que compõe o significado em questão (intensão). A enumeração de membros prototípicos de uma categoria é, aliás, uma excelente ferramenta para ajudar o consulente a entender o significado da unidade léxica definida.

Por fim, citamos um último exemplo bastante interessante da representação da prototipicidade nas whole-sentence definitions:

animal [...] 1 An animal is a living creature such as a dog, lion, or rabbit, rather than a bird, fish, insect, or human being. (CCLD, 2003, s.v.)

Notemos que, nesse caso, o redator da definição opta por apresentar não apenas os membros mais típicos pertencentes à categoria animal (nesta acepção), mas também cita os membros que não pertencem à referida categoria. Esse foi um recurso interessante usado pelo redator, para deixar bem clara a diferença entre esta e as demais acepções de animal. ${ }^{13}$

\subsubsection{Identificação dos traços mais prototípicos da categoria}

Como dissemos anteriormente, o efeito de prototipicidade também pode manifestar-se nas whole-sentence definitions através da identificação dos traços mais prototípicos da categoria. Estes traços aos quais Geeraerts (2001) alude são de natureza extralinguística, estando relacionados com o referente, não com o significado.

Em Farias (2006a e 2008), reconhecem-se dois tipos de informações extralinguísticas: os virtuemas, definidos, de acordo com Pottier (1977), como 
características frequentemente verdadeiras, mas não distintivas, relacionadas ao conhecimento dos indivíduos e às informações enciclopédicas, ou enciclopedismos. Essa distinção ficará mais clara com a análise exposta a seguir.

\subsubsection{Indicação de elementos virtuemáticos}

Transcrevemos abaixo duas definições que apresentam elementos virtuemáticos:

bird [...] $1 \mathrm{~A}$ bird is a creature with feathers and wings. Female birds lay eggs. Most birds can fly. (CCLD, 2003, s.v.)

house [...] 1 A house is a building in which people live, usually the people belonging to one family. (CCLD, 2003, s.v.)

No primeiro caso, o terceiro período que compõe a definição de bird oferecida por CCLD (2003, s.v.) apresenta um elemento virtuemático: embora a experiência nos demonstre que "voar" é uma das características pertinentes ao grupo das aves, e talvez até uma das mais prototípicas, é preciso admitir que, por um lado, nem todos os membros pertencentes à categoria aves podem voar (por exemplo, o pinguim), e, por outro lado, alguns animais que não se incluem nesta classe também apresentam esta propriedade, a exemplo de alguns insetos e de mamíferos como os morcegos. Este traço virtuemático é reconhecido pelo redator da definição, que usa a expressão "a maioria de" [most $]$ ao introduzir esse elemento. Salientamos, no entanto, que, neste caso, para auxiliar o consulente a entender o que é bird, seria mais interessante apresentar uma enumeração dos membros mais típicos da categoria do que listar características dessa classe de animais.

Na definição de house, por sua vez, o redator usa um virtuema que ele próprio reconhece como tal ao introduzi-lo através do advérbio "normalmente" [usually]. Como no caso anterior, sabemos que a condição de que as pessoas que vivem em uma mesma casa pertençam a uma mesma família nem sempre se verifica, muito embora seja frequentemente verdadeira e prototípica de acordo com nosso conhecimento de mundo compartilhado. Notemos, no entanto, que a explicitação desse elemento virtuemático na definição de house, ao contrário do que acontece s.v. bird, não é muito útil para que o leitor entenda o que é house, podendo, dessa forma, ser tranquilamente suprimido da definição. 


\subsubsection{Indicação de informações enciclopédicas}

Apresentamos, a seguir, um bom exemplo de whole-sentence definition que expressa o efeito de prototipicidade através de enciclopedismos:

soap [...] 1 Soap is a substance that you use with water for washing yourself or sometimes for washing clothes. (CCLD, 2003, s.v.)

As informações extralinguísticas apresentadas na definição de soap em $\operatorname{CCLD}(2003$, s.v. ) são altamente pertinentes, na medida em que a prototipicidade das características arroladas é capaz de ajudar o consulente a compreender o que é soap. Isso, no entanto, não é o que ocorre na maioria dos casos em que o dicionário apresenta informações enciclopédicas nas definições:

$\operatorname{dog}[\ldots] \mathbf{1} \mathrm{Adog}$ is a very common four-legged animal that is often kept by people as a pet or to guard or hunt. There are many different breeds of dog. (CCLD, 2003, s.v.)

milk [...] 1 Milk is the white liquid produced by cows, goats, and some other animals, which people drink and use to make butter, cheese, and yoghurt. (CCLD, 2003, s.v.)

water $[\ldots] 1$ Water is a clear thin liquid that has no colour or taste when it is pure. It falls from clouds as rain and enters rivers and seas. All animals and people need water in order to live. (CCLD, 2003, s.v.)

A primeira parte da definição de $\operatorname{dog}$ em $\operatorname{CCLD}(2003$, s.v.) apresenta dois elementos de caráter enciclopédico separados por uma conjunção alternativa, a saber, "criados como animais de estimação" ou "criados para a guarda ou caça", que, embora não sejam essenciais para a compreensão do significado da unidade léxica definida, podem ser usados como auxiliares nessa tarefa. A segunda parte da definição, no entanto, acrescenta uma informação enciclopédica completamente supérflua, que poderia ser eliminada sem dano algum para o consulente.

S.v. milk, no mesmo dicionário, encontramos uma informação de caráter enciclopédico, "produzido por vacas, cabras e alguns outros animais", que, de forma similar ao que ocorre na definição de soap, é altamente pertinente para a compreensão do significado da unidade definida, dado que constitui uma 
característica prototípica do seu referente no mundo. A segunda informação enciclopédica apresentada, "que as pessoas bebem e usam para fazer manteiga, queijo e iogurte", por outro lado, não é relevante na tarefa de facilitar a compreensão para o consulente.

Finalmente, s.v. water em CCLD (2003), o primeiro período corresponde à definição propriamente dita, ao passo que, nos dois períodos seguintes, são apresentadas informações enciclopédicas desnecessárias que, por não auxiliarem o leitor a entender o que é water, poderiam ser descartadas sem prejuízo para a inteligibilidade da definição.

\subsection{As falhas e limitações das whole-sentence definitions com relação à representação da prototipicidade}

Além dos problemas concernentes à presença de elementos prototípicos nas whole-sentence definitions, que expusemos e discutimos mais especificamente em 2.1.2., trataremos, a partir de agora, de alguns casos em que essa característica não é verificada na redação dessas definições. A ausência da representação da prototipicidade pode ser consequência tanto de uma falha na redação quanto de uma limitação da própria técnica definitória.

É possível encontrar, nos dois dicionários analisados, vários exemplos de definições em que a prototipicidade não está representada. Nestes casos, as whole-sentence definitions normalmente se assemelham às paráfrases por genus proximum + differentiae specificae. Contudo, há situações mais graves, nas quais o dicionário não oferece definições propriamente, mas sinônimos. A seguir, apresentaremos e discutiremos alguns desses casos.

\subsubsection{Casos em que as whole-sentence definitions assemelham-se às paráfrases por genus proximum + differentiae specificae}

Não faltam exemplos, nos dicionários analisados, de whole-sentence definitions cuja forma é semelhante à das paráfrases por genus proximum + differentiae specificae. Para exemplificar, confrontamos duas definições de insect, retiradas, respectivamente, de CCLD (2003) e de CcLD (2004): 
insect $[\ldots]$ An insect is a small animal that has six legs. Most insects have wings. Ants, flies, butterflies, and beetles are all insects. (CCLD, 2003, s.v.)

insect $[\ldots]$ An insect is a small creature whit six legs. Most insects have wings. (CcLD, 2004, s.v.)

Ambas as definições compreendem pelo menos duas partes claramente distinguíveis: na primeira, apresentam uma paráfrase que enumera os traços intrínsecos à categoria definida, e, na segunda, apresentam um elemento virtuemático, que, neste caso, aliás, poderia ser dispensado. Além dessas duas partes, a definição oferecida em CCLD (2003, s.v.insect) ainda apresenta uma terceira parte de caráter extensional, em que são listados os membros mais prototípicos da categoria. Esse terceiro elemento acrescido à definição é bastante importante para ajudar o consulente a entender o que é insect, podendo, inclusive, em se tratando da técnica de redação em questão, corresponder à definição propriamente dita. O fato de que CCLD (2003, s.v. insect) apresente este elemento prototípico com sucesso na definição põe em destaque a falha detectada em CcLD (2004, s.v. insect), que omite justamente esta que seria a parte mais importante da definição. De forma similar ao que ocorre s.v. insect em CcLD (2004), há muitos outros exemplos de whole-sentence definitions que, em condições de oferecê-lo, omitem o elemento prototípico, apresentando uma definição que pouco difere de uma paráfrase por genus proximum + differentiae specificae. Temos, a seguir, alguns casos:

bird [...] $1 \mathrm{~A}$ bird is a creature with feathers and wings. Female birds lay eggs. Most birds can fly. (CCLD, 2003, s.v. $)^{14}$

building [...] A building is a structure with a roof and walls. (CcLD, 2004, s.v.)

fruit [...] 1 Fruit or a fruit is something which grows on a tree or bush and which contains seeds or a stone covered by a substance that you can eat. (CCLD, 2003, s.v.)

plant $[\ldots] 1 \mathrm{~A}$ plant is a living thing that grows in the earth and has stem, leaves, and roots. (CCLD, 2003, s.v.) 
Há outros casos, porém, em que é mais difícil, se não impossível, expressar a prototipicidade na whole-sentence definition, seja sob a forma de enumeração dos membros mais típicos - o que vai naturalmente se tornando cada vez mais complicado, na medida em que nos encontramos com unidades léxicas mais hiponímicas -, seja sob a forma de indicação dos traços mais prototípicos. Encaixam-se no grupo de palavras cuja definição através dessa técnica é menos eficaz os realia ${ }^{15}$ e as unidades léxicas que fazem parte de nomenclaturas, ambos lembrados por Landau (2001, p. 180). A tentativa de definir os itens léxicos pertencentes a um dos grupos mencionados reflete uma das limitações da técnica definitória em questão. As paráfrases transcritas a seguir ilustram esse problema. Notemos que, salvo pelo fato de que a palavra-entrada está repetida nas definições, elas apresentam traços que as aproximam mais das paráfrases por genus proximum + differentiae specificae do que das whole-sentence definitions, considerando as características destas expostas anteriormente:

boy [...] 1 A boy is a male child. (CcLD, 2004, s.v.)

child [...] 1 A child is a human being who is not yet an adult. (CcLD, 2004, s.v.)

$\operatorname{man}[\ldots] 1 \mathrm{~A}$ man is an adult male human being. (CCLD, 2003, s.v.)

woman [...] 1 A woman is an adult female human being. (CCLD, 2003, s.v.)

bachelor [...] A bachelor is a man who has never married. (CCLD, 2003, s.v.)

love affair [...] A love affair is a romantic and usually sexual relationship between two people. (CcLD, 2004, s.v.)

theory $[\ldots] 1 \mathrm{~A}$ theory is a formal idea or set of ideas that is intended to explain something. (CCLD, 2003, s.v.)

wind $[\ldots] 1 \mathrm{~A}$ wind is a current of air that is moving across the earth's surface. (CCLD, 2003, s.v.)

zoology $[. .$.$] Zoology is the scientific study of animals. (CCLD,$ 2003, s.v.) 


\subsubsection{Casos em que as whole-sentence definitions assemelham-se a uma definição sinonímica}

De acordo com Martínez de Souza (1995), definição sinonímica é aquela na qual a palavra-entrada é definida por um sinônimo ou, ainda, aquela em que se usam palavras pertencentes à mesma família do signo-lema (cf. MARTÍNEZ DE SOUZA, 1995, s.v. definición por sinónimos). A definição através de um sinônimo não constitui um problema em si, mas é preciso considerar que se espera, em um dicionário semasiológico, que o comentário semântico apareça representado por uma paráfrase definidora. Autilização de um sinônimo como um recurso de viés semasiológico, no entanto, poderia justificar-se pelo fato de o sinônimo apresentado ser mais elucidativo do que uma definição ${ }^{16}$ (cf. BUGUEÑO, 2007b e BUGUEÑO; FARIAS, 2007a). Assim sendo, o condenável numa solução sinonímica para o comentário semântico é a alternância indiscriminada entre sinônimos e paráfrases definidoras, o que evidencia a falta ou o não cumprimento de um programa de informações microestruturais. Além disso, é preciso recordar que esse tipo de definição pode acabar gerando o problema chamado círculo vicioso na definição (cf. MARTÍNEZ DE SOUZA, 1995, s.v. definición lingüística), também conhecido como circularidade (cf. LANDAU, 2001, p. 157-160) ${ }^{17}$.

Apresentamos, a seguir, um exemplo de whole-sentence definition que se assemelha a uma definição sinonímica e que, consequentemente, pode acarretar problemas idênticos aos desta:

twister [...] A twister is the same as a tornado. (AM) (CCLD 2003, s.v.)

O sinônimo apresentado nesse caso específico pode ser uma boa opção se pensamos exclusivamente no usuário brasileiro aprendiz de inglês. É necessário, no entanto, lembrar que CCLD (2003) é um dicionário monolíngue de inglês destinado a aprendizes desta língua em geral, sem discriminar um público específico. Assim sendo, se pensamos, por exemplo, num usuário japonês, uma definição desse tipo poderia não ser uma boa opção, já que obrigaria o leitor a realizar uma nova busca no dicionário, desta vez, por tornado, a fim de tentar encontrar o significado de twister, palavra que motivou a primeira consulta. Há, contudo, casos mais problemáticos que este: 
idea [...] 1 An idea is a plan, suggestion, or possible course of action. (CCLD, 2003, s.v.)

Nesse caso, se o usuário deseja saber o significado de idea, necessitará realizar três consultas adicionais, buscando, respectivamente, plan, suggestion e course. Esse problema evidencia uma falha gritante no processo de redação das definições.

\section{Contraste entre as whole-sentence definitions e as paráfrases por genus proximum + differentiae specificae}

Até agora, detivemo-nos na análise das whole-sentence definitions, procurando elencar as características mais marcantes desta técnica definitória, bem como identificar as possíveis falhas, tanto de método quanto de redação. Nesta seção, aproveitaremos o material obtido através da análise realizada, a fim de confrontar a técnica das definições sentenciais com a das paráfrases por genus proximum + differentiae specificae. Nossa comparação será dividida em dois momentos distintos: no primeiro, e mais breve, retomaremos as aproximações das whole-sentence definitions às paráfrases tradicionais, que já discutimos em 2.2.; no segundo, trataremos dos casos em que as definições por genus proximum + differentiae specificae também apresentam elementos prototípicos.

\subsection{Ausência da representação da prototipicidade nas whole- sentence definitions}

Como vimos na seção anterior, uma das características das wholesentence definitions é utilizar-se de um elemento prototípico na definição (cf., por exemplo, as definições de clothes, bird e soap, transcritas anteriormente). Entretanto, em muitos casos, esse elemento é utilizado de maneira pouco proveitosa (cf., por exemplo, as definições de house e water, transcritas anteriormente), ou, ainda, está ausente nas paráfrases. A ausência da representação da prototipicidade nas definições, por sua vez, pode decorrer, como vimos, de uma falha no momento da redação (cf., por exemplo, as definições de fruit e plant, transcritas anteriormente), ou de uma limitação da própria técnica definitória (cf., por exemplo, as definições de man, woman e child, transcritas anteriormente). Nesta última situação, não resta outra saída ao lexicógrafo que 
recorrer a um termo hiperonímico acompanhado de um ou mais especificadores para a redação da definição. Assim, a solução que CCLD (2003, s.v. banana) encontra para definir a unidade léxica em questão é usar o hiperônimo "fruit", acompanhado dos especificadores "curved", "long" e "with yellow skins":

banana [...] 1 Bananas are long curved fruit with yellow skins. (CCLD, 2003, s.v.)

Como vemos, salvo pelo fato de que a palavra-entrada é repetida na definição, não há nenhuma outra diferença entre esta e uma paráfrase tradicional por genus proximum + differentiae specificae. Observemos, a título de ilustração, a semelhança entre a definição transcrita acima, e a paráfrase oferecida em OALD (2005):

banana $[. .$.$] a long curved fruit with a thick yellow skin and soft$ flesh, that grows on trees in hot countries. (OALD, 2005, s.v.)

Essa definição seria praticamente idêntica à de CCLD (2003, s.v. banana), não fosse pelo fato de que OALD (2005, s.v. banana) usa um elemento de caráter enciclopédico, "that grows on trees in hot countries", que não contribui em nada para a compreensão do significado da unidade léxica definida. Aliás, o uso de enumerações e de elementos de caráter enciclopédico e virtuemático em dicionários que optam pela técnica das paráfrases por genus proximum + differentiae specificae será tema do nosso próximo tópico.

\subsection{A representação da prototipicidade nas paráfrases por genus proximum + differentiae specificae}

De acordo com Geeraerts (2001), "[w]ithin a structuralist conception of semantics, this [i.e. o uso de elementos prototípicos nas definições] would be inadmissible, because these elements belong to the 'encyclopedic' level rather than the semantic level"' (GEERAERTS, 2001, p. 14). Entretanto, como o próprio autor reconhece a seguir, essa prática é cada vez mais comum, e é preciso admitir que, muitas vezes, o uso de enumerações, bem como de elementos virtuemáticos e enciclopédicos nas definições pode ser uma valiosa ferramenta de auxílio à compreensão do significado. A seguir, apresentamos alguns exemplos da 
introdução destes três tipos de informações em paráfrases por genus proximum + differentiae specificae. Com isso, queremos deixar claro que, ao contrário do que a análise efetuada na seção 2 deste trabalho possa induzir a pensar, essa característica não é exclusiva das whole-sentence definitions.

\subsubsection{A presença de enumerações de membros da categoria definida nas paráfrases por genus proximum + differentiae specificae}

A teoria metalexicográfica estabelece uma distinção entre os princípios, e também entre as técnicas, para a representação da intensão e da extensão nas definições (cf. 2.1.1.). As definições formuladas sob o princípio de genus proximum + differentiae specificae, como vimos, são definições intensionais. Contudo, muitas vezes, o lexicógrafo julga que uma paráfrase desse tipo não é suficiente para ajudar o leitor a entender o significado da palavra-entrada. Por essa razão, em alguns casos, introduz-se, dentro da definição intensional, um elemento de caráter extensional, representado por uma enumeração dos membros mais prototípicos da categoria definida.

Essa solução que o dicionário encontra ajuda o consulente não somente a entender o que a palavra significa, mas também a saber a quais referentes extralinguísticos esse conceito se aplica (cf. GEERAERTS, 2003, p. 88-91). Abaixo, citamos alguns exemplos dessa prática em dicionários que optam pela técnica tradicional de definição: ${ }^{18}$

baga $[\ldots] 1[\ldots]$ fruto simples, carnoso, indeiscente, freq. [frequentemente] comestível, com um ou mais carpelos e sementes (p.ex., tomate, uva, mamão, goiaba etc.) (Hou, 2001, s.v.)

roedores $[\ldots] 1$ 1. Ordem de mamíferos terrestres e fossórios, ocasionalmente arborícolas ou semiaquáticos, de pés unguiculados e 22 dentes. $\mathrm{O}$ esmalte dos incisivos superiores não alcança a superfície interna, provocando-lhes o crescimento contínuo. $\underline{\text { São os }}$ esquilos, os ratos, os ouriços e as preás. (Au, 1999, s.v.)

Droga blanda. Estupefaciente que no es adictivo o lo es en bajo grado; como el hachís o la marihuana. (DUE, 2001, s.v. droga) 
D. [droga] dura. Estupefaciente muy adictivo que puede causar trastornos graves; como la cocaína o la heroína. (DUE, 2001, s.v. droga)

pomo [...] $1 \mathrm{~m}$. Fruto; como la manzana o la pera, que tiene el mesocarpio carnoso, y, en su interior, varias celdillas de tejido cartilaginoso en las que están encerradas las semillas. (DUE, 2001, s.v.)

mammal [...] any animal that gives birth to live babies, not eggs, and feeds its young on milk. Cows, humans and wHALES are all mammals. (OALD, 2005, s.v.)

\subsubsection{A presença de informações enciclopédicas e virtuemáticas nas paráfrases por genus proximum + differentiae specificae}

Em Farias (2006a e 2008), procurou-se demonstrar que nem sempre é possível formular paráfrases definidoras que respeitem rigorosamente o critério de utilizar apenas semas como especificadores. Assim sendo, os virtuemas e os enciclopedismos podem vir a ser usados como especificadores na redação das paráfrases por genus proximum + differentiae specificae, mas é preciso saber como e quando utilizá-los de forma proveitosa. Para tanto, estabeleceu-se que os elementos enciclopédicos e virtuemáticos somente devem estar presentes na formulação das definições se, de fato, constituem informações relevantes para a compreensão do significado e são de fácil inferência para o consulente. A seguir, listamos algumas definições que estão formuladas de acordo com esse princípio: ${ }^{19}$

formão [...] 1 ferramenta manual, própria para madeira, com uma extremidade embutida num cabo e a outra chata, terminando em lâmina afiada [Usado esp. [especialmente] em obras de talha e corte de ensambladuras.] (Hou, 2001, s.v.)

inodoro $[\ldots] 2$ [...] Recipiente con una cisterna de agua en el que se orina y se hace de vientre. (Señas, 2002, s.v.) 
muleta [...] 3 Taurom. Palo con un paño rojo sujeto a él por una de sus orillas, con el que el torero trastea al toro. (DUE, 2001, s.v.)

cigarette $[. .$.$] a thin tube of paper filled with товАСС, for smoking.$ (OALD, 2005, s.v.)

As informações enciclopédicas contidas nessas quatro definições cumprem um papel importante no que diz respeito à compreensão do significado. Notemos que, em especial, s.v. inodoro, em Señas (2002), e s.v. muleta, em DUE (2001), o elemento sublinhado constitui a parte mais importante da definição, sem a qual o significado não seria preciso. Destacamos, ainda, o procedimento de Hou (2001, s.v. formão), que separa a informação enciclopédica da definição propriamente dita, através do uso de colchetes. Como esta é uma informação suplementar, acreditamos ser muito acertada a decisão de separá-la do conteúdo semântico principal. ${ }^{20}$

No entanto, na maioria das vezes, as informações virtuemáticas e enciclopédicas não são utilizadas de maneira proveitosa nos dicionários. Grande parte dos enciclopedismos apresentados, por exemplo, são informações completamente desnecessárias, que, além de não auxiliarem o leitor a compreender o significado da palavra definida, podem, em muitos casos, confundi-lo. A seguir, transcrevemos algumas definições problemáticas encontradas nos dicionários: ${ }^{21}$

abacaxi ${ }^{1}[$...] 1. Planta da família das bromeliáceas (Ananas sativus), cultivada ou selvagem, cuja parte comestível é infrutescência carnosa resultante do crescimento e da coalescência de todas as flores da inflorescência. Tanto a infrutescência como o caule encerram uma enzima proteolítica que pode ter o mesmo emprego que a papaína. ( $\mathrm{Au}, 1999$, s.v.)

avestruz [...] 1 [...] ave da fam. [família] dos estrutionídeos (Struthio camelus), encontrada nas planícies áridas da África e Arábia, com cerca de 2,5 $\mathrm{m}$ de altura, pernas longas e fortes, pés com apenas dois dedos, cabeça e pescoço praticamente implumes [É a maior ave vivente.] (Hou, 2001, s.v.)

melancia [...] 1. Planta herbácea, prostrada, da família das cucurbitáceas (Citrullus vulgaris), de origem africana, de folhas 
bastante subdivididas, e cultivada por causa dos frutos, enormes bagas uniloculares e polispermas, muito sucosas, de casca verde e polpa vermelha com sementes negras; melancieira, balancia. (Au, 1999, s.v.)

carpa $^{1}[\ldots]$ 1. f. Pez teleósteo fisóstomo, verdoso por encima y amarillo por abajo, de boca pequeña sin dientes, escamas grandes y una sola aleta dorsal, que vive muchos años en las aguas dulces. Hay una especie procedente de China, de color rojo y dorado. (DRAE, 2001, s.v.)

fruit $[\ldots] 3$ [...]Fruit $\left(2^{\circ}\right)$ comestible, lorsqu'il est sucré, que l'on consomme généralement au dessert, parfois comme accompagnement (canard à l'orange, porc aux pruneaux, dinde aux marrons, etc.). (PRb, 2001, s.v.)

oranger [...] Arbre fruitier (rutacées), au feuillage luisant, persistant et parfumé, originaire de Chine, qui produit les oranges. (PRb, 2001, s.v.)

\section{Conclusões}

Ao longo da exposição realizada, tentamos identificar as principais características, bem como as limitações das whole-sentence definitions, sempre procurando contrapô-las às paráfrases por genus proximum + differentiae specificae.

Primeiramente, identificamos duas características fundamentais das whole-sentence definitions: a) o período definitório encontra-se normalmente estruturado em duas orações - a primeira mostra a palavra-entrada em um contexto, e a segunda apresenta a definição propriamente tal - e b) a paráfrase definidora geralmente apresenta um viés extensional, seja pela indicação do efeito de sentido provocado pelo uso da unidade léxica definida (cf., por exemplo, as definições de cute e polite, apresentadas anteriormente), seja sob a forma de um elemento prototípico na definição.

Em segundo lugar, a análise que realizamos na seção 2 permitiu-nos concluir que a técnica em questão apresenta duas restrições básicas: 
1) No que diz respeito à representação da prototipicidade através da enumeração de membros da categoria definida, quanto mais hiperonímica for a unidade léxica definida, mais fácil será gerar definições que apresentem um elemento prototípico. Consequentemente, quanto mais específica for a unidade definida, mais improvável será gerar whole-sentence definitions com essa característica. Comparemos, por exemplo, a definição de human being "A human being is a man, woman, or child" com as definições de man "A man is an adult male human being" e de woman "A woman is an adult female human being", todas as três apresentadas em CCLD (2003, s.v.).

2) No que diz respeito à representação da prototipicidade através da indicação de elementos virtuemáticos e enciclopédicos, o sucesso desse procedimento esbarra nas limitações que a língua impõe, dado que algumas palavras, por sua própria natureza, são muito difíceis de definir. Landau (2001,p. 180), por exemplo, aponta as unidades pertencentes a nomenclaturas e os realia como itens com os quais as whole-sentence definitions não funcionam bem. A constatação de Landau (2001) coincide, em parte, com os resultados obtidos em Beneduzi, Bugueño e Farias (2005), que apontam os nomes de plantas, frutos e animais, bem como as unidades léxicas que se incluem em taxonomias fechadas como palavras complicadas de definir, mesmo tratando-se de definições tradicionais.

Por fim, o contraste com as paráfrases por genus proximum + differentia specificae permitiu-nos responder à seguinte pergunta: em que, afinal, as wholesentence definitions diferem das paráfrases tradicionais? Na verdade, as diferenças entre as duas técnicas definitórias, pelo menos no que diz respeito à representação da prototipicidade nas definições dos substantivos, são menores do que poderíamos supor a princípio. O cotejo entre os dois procedimentos definitórios revela que, se por um lado, as whole-sentence definitions nem sempre contemplam a apresentação de elementos prototípicos na sua redação, por outro lado, esse tipo de informação, como vimos, cada vez mais vem ganhando espaço nas paráfrases por genus proximum + differentiae specificae. Isso significa que, pelo menos no que diz respeito à definição dos substantivos, salvo pelo fato de que as whole-sentence definitions se apresentam formalmente como uma sentença completa, não há uma grande diferença entre o resultado final obtido pelo emprego de uma ou de outra técnica. 


\section{Notas}

${ }^{1}$ A distinção entre semasiologia e onomasiologia é essencial para a Lexicografia, permitindo gerar uma oposição entre os dicionários semasiológicos e os dicionários onomasiológicos (cf., por exemplo, HARTMANN; JAMES, 2001, s.v. onomasiology, semasiology e GEERAERTS, 2003, p. 83-84). Os dicionários semasiológicos têm como principal característica apresentar paráfrases definidoras (cf. HARTMANN; JAMES, 2001, s.v. semasiological dictionary). Os dicionários onomasiológicos, por sua vez, caracterizam-se pelo estabelecimento de relações conceituais ou de sentido entre as palavras, sendo exemplos de dicionários onomasiológicos os thesaurus e os dicionários de sinônimos e antônimos (cf. HARTMANN; JAMES, 2001, s.v. onomasiological dictionary).

${ }^{2}$ Macro e microestrutura são os níveis fundamentais de estruturação de um dicionário (cf. HARTMANN; JAMES, 2001, s.v. structure). São concernentes ao âmbito macroestrutural todas as questões relacionadas com o estabelecimento do número de unidades léxicas arroladas, com o tipo de unidades registradas e com a disposição lemática (a esse respeito, cf. HAENSCH, 1982, p. 452-457; HAUSMANN; WIEGAND, 1989; HARTMANN, 2001, p. 64; HARTMANN; JAMES, 2001, s.v. macrostructure, LANDAU, 2001, p. 99 e ss.; WELKER, 2004, p.80-107; BUGUEÑO, 2001, 2005 e 2007a e BUGUEÑO; FARIAS, 2007b). Por sua vez, dizem respeito ao âmbito microestrutural todos os problemas relacionados com a determinação do programa constante de informações e a organização interna dos verbetes (a esse respeito, cf. DUBOIS; DUBOIS, 1971, p. 39-46; MERZAGORA, 1987, p. 107-124; HAUSMANN; WIEGAND, 1989; HARTMANN, 2001, p. 64-65; HARTMANN; JAMES, 2001, s.v. comment, microstructure; WELKER, 2004, p. 107-177; GARRIGA, 2003, p. 105-126; BUGUEÑO, 2004 e BUGUEÑO; FARIAS, 2006 e 2007a).

${ }^{3}$ Martínez de Souza (1995), por exemplo, apresenta em seu dicionário nada menos que 46 entradas para o tema "definição", tendo em vista, além das diferentes opções metodológicas disponíveis, a variedade terminológica existente.

${ }^{4} \mathrm{O}$ primeiro parâmetro leva em conta o ponto de partida do ato da consulta (o significante ou o significado), o que implica a adoção de uma perspectiva semasiológica ou onomasiológica respectivamente (cf. nota 1). O segundo parâmetro baseia-se na distinção proposta por Seco (1987) entre metalinguagem de primeiro enunciado e metalinguagem de segundo enunciado. Enquanto as paráfrases formuladas em metalinguagem de segundo enunciado (ou metalinguagem de conteúdo) exprimem o conteúdo semântico da unidade definida, as paráfrases em metalinguagem de primeiro enunciado (ou metalinguagem de signo) explicam como a palavra é empregada. 
${ }^{5}$ Seguindo a tendência da metalexicografia europeia, identificaremos todos os dicionários citados neste trabalho através de abreviaturas (cf. HARTMANN, 2001, p. 11).

${ }^{6}$ Para uma visão geral do projeto do dicionário de falsos amigos espanhol-português, cf. Bugueño (2003).

${ }^{7}$ Em Martínez de Souza (1995, s.v. definición lingüística), entende-se circularidade como a propriedade de intercambiar a unidade léxica definida pela sua definição em um dado contexto, sem que haja alteração de significado. Landau (2001, p. 157-160), por outro lado, designa por circularidade um vício de definição que consiste em parafrasear a unidade A em termos de B, e a unidade B em termos de A, ou ainda definir A em termos de A. Esse vício, por sua vez, é identificado em Martínez de Souza (1995, s.v. definición lingüística) como círculo vicioso na definição.

${ }^{8}$ Tanto Landau (2001, p. 164-166), quanto Seco (1987, p. 22-23), reconhecem que a prova da substituição, apesar de figurar como um dos princípios fundamentais do bem definir, além de não ser característica de todos os tipos de definição, como é o caso das whole-sentence definitions, bem como da chamada when-definition (cf. LEW; DZIEMIANKO, 2006a e 2006b) ou folk definition (cf. HARTMANN; JAMES, 2001, s.v.), tampouco pode ser aplicada indiscriminadamente a todas as classes de palavras. Esse é o caso do grupo de palavras que Borba (2003, p. 45-46) chama de gramaticais, tais como os pronomes, as conjunções e as preposições.

${ }^{9} \mathrm{O}$ modelo das condições necessárias e suficientes, também conhecido como teoria clássica de categorização, sustenta-se nos seguintes princípios: 1) as categorias são entendidas como possuidoras de fronteiras claramente delimitadas; 2) a pertinência de uma entidade particular a uma dada categoria depende do cumprimento das condições criteriais da mesma; 3) todos os membros têm igual representatividade dentro de uma categoria. Sobre o modelo das condições necessárias e suficientes, cf. Kleiber (1990, p. 21-43).

${ }^{10}$ Para uma introdução à Teoria dos Protótipos, cf. Kleiber (1990) e Evans e Green (2006, p. 27-50).

${ }^{11}$ Para uma introdução ao problema da intensão e da extensão nas definições, cf. Schifko (1992, p. 136), Martínez de Souza (1995, s.v. definición extensional, definición intensional), Hartmann e James (2001, s.v. extensional definition, intensional definition) e Geeraerts (2001 e 2003, p. 88-91).

${ }^{12} \mathrm{O}$ caráter extensional, no entanto, não é uma característica exclusiva das wholesentence definitions, podendo manifestar-se, igualmente, em paráfrases por genus proximum + differentiae specificae, como veremos em 3.2. 
${ }^{13} \mathrm{O}$ dicionário arrola outras duas acepções para animal como substantivo, a saber, "Any living creature other than a human being can be referred to as an animal" e "Any living creature, including a human being, can be referred to as an animal" (cf. CCLD 2003, s.v., ac. 2 e 3).

${ }^{14}$ Para uma discussão acerca dos problemas específicos dessa definição, cf. 2.1.2.1.

${ }^{15}$ Realia é um termo de uma dada língua que designa uma realidade particular de uma cultura. São exemplos de realia, no português, bumba-meu-boi "dança do folclore brasileiro" e feijoada "iguaria preparada com feijão"; no inglês, green card "documento que permite a permanência de um estrangeiro nos Estados Unidos"; no alemão, Abitur "exame final do ensino secundário necessário para admissão no ensino superior" e Keiserschmarren "espécie de panqueca polvilhada com açúcar, típica da Áustria, Suíça e sul da Alemanha"; no espanhol, falla "figura gigante que se queima durante os festejos de São José na região de Valência" e gazpacho "espécie de sopa fria".

${ }^{16}$ Comparemos, por exemplo, as paráfrases de deglutir apresentadas respectivamente em Au (1999) e Hou (2001): "Engolir, ingerir" (Au, 1999, s.v. deglutir, ac. 1) e "passar (o bolo alimentar) da boca para o esôfago e, a seguir, para o estômago" (Hou, 2001, s.v. deglutir).

${ }^{17}$ A esse respeito, cf. nota 7. Problemas dessa classe, infelizmente, são bem comuns em dicionários semasiológicos. Um bom exemplo de círculo vicioso na definição pode ser encontrado em MiMe (2000): castigo "Pena, punição" (MiMe, 2000, s.v., ac. 2), pena " "Castigo, punição" (MiMe, 2000, s.v., ac. 1) e punição "Pena, castigo" (MiMe, 2000, s.v.). Mais exemplos desse problema foram detectados na análise de um dicionário escolar de língua espanhola (cf. FARIAS, 2006b).

${ }^{18}$ Todos os grifos são nossos.

${ }^{19}$ Todos os grifos são nossos.

${ }^{20}$ Esse procedimento foi adotado na redação das paráfrases definidoras do dicionário de falsos amigos espanhol-português. No referido dicionário, utilizou-se o recurso formal dos parênteses para separar as informações virtuemáticas e enciclopédicas, que foram chamadas de incrementos contextuais, do conteúdo principal da definição (cf. BENEDUZI; BUGUEÑO; FARIAS, 2005).

${ }^{21}$ Todos os grifos são nossos. 


\section{Referências}

Au (1999). FERREIRA, A. B. de H. Novo Aurélio século XXI: O dicionário da língua portuguesa. Rio de Janeiro: Nova Fronteira. 1999. 1 CD-ROM.

BENEDUZI, R. Análise das definições em quatro dicionários semasiológicos da língua portuguesa e propostas de emendas. Ao Pé da Letra 6, p. 183-190, 2004. BENEDUZI, R.; BUGUEÑO, F. Aprendendo a ler um dicionário: análise de verbetes substantivos. Revista Língua \& Literatura 6/7, p. 113-122, 2005.

BENEDUZI, R.; BUGUEÑO, F.; FARIAS, V. Avanços na redação de um dicionário de falsos amigos espanhol-português. Lusorama 61/62, p. 195-219, 2005.

BORBA, F. Organização dos dicionários. Uma introdução à lexicografia. São Paulo: Universidade Estadual Paulista, 2003. 356p.

BUGUEÑO, F. Problemas macroestruturais em dicionários de falsos amigos. Expressão 5, p. 89-93, 2001.

BUGUEÑO, F. Consideraciones para un nuevo diccionario de falsos amigos españolportugués. Polifonia 6, p. 103-127, 2003.

BUGUEÑO, F. Notícia sobre o comentário de forma e o comentário semântico em um dicionário de falsos amigos espanhol-português. Expressão 8, p. 89-93, 2004.

BUGUEÑO, F. O que o professor deve saber sobre a nominata do dicionário de língua. Revista Língua \& Literatura 6/7, p. 17-31, 2005.

BUGUEÑO, F. O que é macroestrutura no dicionário de língua? In: ISQUERDO, A. N.; ALVES, I. M. (Org.). As ciências do léxico: lexicologia, lexicografia e terminologia. Campo Grande: Humanitas, 2007a. p. 261-272.

BUGUEÑO, F. Para uma taxonomia de definições. 2007b. [Inédito]

BUGUEÑO, F.; FARIAS, V. S. Informações discretas e discriminantes no artigo léxico. Cadernos de Tradução 18, p. 115-135, 2006.

BUGUEÑO, F.; FARIAS, V. S. Avaliação do programa de informações em dicionários monolíngües de português. In: Congresso Brasileiro de Lingüística Aplicada, 8, 2007, Brasília. Anais. Brasília: Universidade de Brasília, 2007a. [No prelo]

BUGUEÑO, F.; FARIAS, V. S. Desenho da macroestrutura de um dicionário escolar de língua portuguesa. In: Colóquio Internacional de Lexicografia Pedagógica, 1, 2007, Florianópolis. Anais. Florianópolis: Universidade Federal de Santa Catarina, 2007b. [No prelo]

CCLD (2003). Collins Cobuild Advanced Learner's Dictionary. Glasgow: HarperCollins, 2003. 1 CD-ROM. 
CcLD (2004). Collins Cobuild Compact English Learner's Dictionary. Glasgow/ São Paulo: HarperCollins/DISAL, 2004. 726p.

DRAE (2001). Diccionario de la lengua española. Madrid: Espasa-Calpe. 2001. Disponível em: <www.rae.es>. Acesso em: 27/12/2007.

DUBOIS, J.; DUBOIS, C. Introduction à la lexicographie: le dictionnaire. Paris: Larousse, 1971. 224p.

DUE (2001). MOLINER, M. Diccionario de uso del español. Madrid: Gredos. 2001. 1 CD-ROM.

EVANS, V.; GREEN, M. Cognitive Linguistics: An introduction. London: Lawrence Elrbaum, 2006. 811p.

FARIAS, V. S. La presentación del comentario semántico en los diccionarios escolares. Revista de Letras 70, p. 183-205, 2006a.

FARIAS, V. S. Reseña al Diccionario Ilustrado de la Lengua Española (2004). Anuario Brasileño de Estudios Hispánicos 16, p. 199-201, 2006b.

FARIAS, V. S. Considerações sobre a redação das glosas em um dicionário de falsos amigos espanhol-português, 2008. [Inédito]

GARRIGA, C. La microestructura del diccionario: Las informaciones lexicográficas. In: MEDINA GUERRA, A. M. (Org.). Lexicografía española. Barcelona: Ariel, 2003. p.103-126.

GEERAERTS, D. The definitional practice of dictionaries and the Cognitive Semantic conception of polysemy. Lexicographica 17, p. 6-21, 2001.

GEERAERTS, D. Meaning and definition. In: VAN STERKENBURG, P. (Org.). A practical guide to lexicography. Amsterdam/Philadelphia: John Benjamin, 2003. p.83-93.

HAENSCH, G. La lexicografía: de la lingüística teórica a la lexicografía práctica. Madrid: Gredos, 1982. 563p.

HARTMANN, R. R. K. Teaching and researching lexicography. London: Longman, 2001. 211p.

HARTMANN, R. R. K.; JAMES, G. Dictionary of lexicography. London: Routledge, 2001. 176p.

HAUSMANN, F. J.; WIEGAND, H. E. Component Parts and Structures of General Monolingual Dictionaries: A Survey. In: HAUSMANN, F. J.; REICHMANN, O.; WIEGAND, H. E.; ZGUSTA, L. (Org.). Wörterbücher, dictionaries, dictionnaires. Ein internationales Handbuch zur Lexikographie. Berlin/New York: Walter de Gruyter, 1989. p.328-360. 
Hou (2001). HOUAISS, A. Dicionário Houaiss da língua portuguesa. Rio de Janeiro: Objetiva, 2001. 1 CD-ROM.

JACKSON, H. Lexicography. London: Routledge. 2002. 204p.

KLEIBER, G. La sémantique du prototype. Paris: Presses Universitaires de France. 1990. 208p.

LAKOFF, G. Woman, fire and dangerous things: what categories reveal about the mind. Chicago: University of Chicago Press, 1987. 614p.

LANDAU, S. Dictionaries: The art and craft of lexicography. Cambridge: Cambridge University Press, 2001. 494p.

LEW, R.; DZIEMIANKO, A. A new type of folk-inspired definition in English monolingual learner's dictionaries and its usefulness for conveying syntactic information. International Journal of Lexicography 19, p. 225-242, 2006a.

LEW, R.; DZIEMIANKO, A. Non-standard dictionary definitions: what they cannot tell native speakers of Polish. Cadernos de Tradução 18, p. 275-294, 2006b.

MARTÍNEZ DE SOUZA, J. Diccionario de lexicografía práctica. Barcelona: Bibliograf, 1995. 380p.

MERZAGORA, G. M. La lessicografia. Bologna: Zanichelli, 1987. 180p.

MiMe (2000). Melhoramentos: minidicionário escolar da língua portuguesa. São Paulo: Melhoramentos, 2000. 658p.

OALD (2005). Oxford Advanced Learner's Dictionary. Oxford: Oxford University Press, 2005. 1780p.

POTTIER, B. A definição semântica nos dicionários. In: LOBATO, L. M. P. (Org.). A semântica na linguística moderna: o léxico. Rio de Janeiro: Francisco Alves, 1977. p. 21-31.

PRb (2001). Nouveau Petit Robert Dictionnaire alphabétique et analogique de la langue française. Paris: Édition Le Robert, 2001. 1 CD-ROM

SCHIFKO, P. Lexicología y semántica. In: HOLTUS, G.; METZELTIN, M.; SCHMITT, C. (Org.). Lexikon der romanistischen Linguistik. Tübingen: Max Niemeyer, 1992. p.132-148.

SECO, M. Estudios de lexicografía española. Madrid: Paraninfo, 1987. 468p.

Señas (2002). Señas Diccionario para la enseñanza de la lengua española para brasileños. São Paulo: Martins Fontes, 2002. 1510p. 
WELKER, H. A. Dicionários: Uma pequena introdução à lexicografia. Brasília: Thesaurus, 2004. 301p.

ZANATTA, Flávia. La calidad de las definiciones lexicográficas de cuatro diccionarios de americanismos. Anuario Brasileño de Estudios Hispánicos 16, p. 135-152, 2006. 